\title{
Phonon-mode specific contributions to room-temperature superconductivity in atomic hydrogen at high pressures
}

\author{
Ashok K. Verma $\odot^{*}$ and P. Modak $\odot$ \\ High Pressure and Synchrotron Radiation Physics Division, Bhabha Atomic Research Centre, Mumbai 400085, India \\ Fabian Schrodi $\odot,^{\dagger}$ Alex Aperis $\odot, \$$ and Peter M. Oppeneer $\odot$ \\ Department of Physics and Astronomy, Uppsala University, P.O. Box 516, SE-75120 Uppsala, Sweden
}

(Received 23 November 2020; revised 25 February 2021; accepted 26 February 2021; published 10 March 2021)

\begin{abstract}
We investigate the role of specific phonon mode symmetries for the room-temperature superconductivity in atomic hydrogen under large pressure. Using anisotropic Migdal-Eliashberg theory with $a b$ initio input from density functional theory, we show that the $E_{u}$ phonon modes are the dominant driving force for obtaining such high critical temperatures. When going from 400 to $600 \mathrm{GPa}$, we find an increased transition temperature; however, the total electron-phonon coupling strength is counterintuitively reduced. Our analysis reveals that this is due to an enhanced contribution to the coupling strength by the $E_{u}$ phonon mode. We furthermore compute the momentum anisotropy of the superconducting gap which we find to be relatively small, about $7 \%$ of the mean gap value at $100 \mathrm{~K}$.
\end{abstract}

DOI: 10.1103/PhysRevB.103.094505

\section{INTRODUCTION}

Reaching superconductivity at room temperature has been the focus of intense research activities in the last few years (see $[1,2]$ for recent surveys). Especially promising results have been achieved for superhydrides, such as $\mathrm{H}_{3} \mathrm{~S}$, with a transition temperature of $203 \mathrm{~K}$ at a pressure of $155 \mathrm{GPa}$ [3], $\mathrm{LaH}_{10}$ with a $T_{c}$ around $250 \mathrm{~K}$ at a pressure of $170 \mathrm{GPa}$ or higher [4-6], and $\mathrm{YH}_{6}$ with $T_{c} \simeq 220 \mathrm{~K}$ at $166-237 \mathrm{GPa}[7,8]$. Very recent studies report room-temperature superconductivity $(287 \mathrm{~K})$ in a carbonaceous sulfur hydride at $267 \mathrm{GPa}$ [9], and possibly even a higher critical temperature in a La superhydride mixed with ammonia borane [10]. A unifying aspect of these recently discovered high-temperature superconductors is the prevalent conventional electron-phonon mechanism that is responsible for the record high critical temperatures [2].

The quest for room-temperature superconductivity in hydrides goes back to a proposal by Ashcroft [11], stating that dense atomic hydrogen metal could exhibit superconductivity at a very high critical temperature. The existence of a metallic phase of atomic hydrogen was first conceived by Wigner and Huntington in 1935 [12]. Since these seeding works, massive efforts have been devoted to experimentally confirm such predictions at high pressures (see $[13,14]$ ), eventually aiming for

\footnotetext{
*hpps@barc.gov.in

†fabian.schrodi@physics.uu.se

‡alex.aperis@physics.uu.se
}

Published by the American Physical Society under the terms of the Creative Commons Attribution 4.0 International license. Further distribution of this work must maintain attribution to the author(s) and the published article's title, journal citation, and DOI. Funded by Bibsam. the final demonstration of high-temperature superconductivity in this material. However, the formation of atomic hydrogen metal at high pressure has been difficult to establish in diamond-anvil pressure cells. So far, some evidence for a metallic phase has been presented at various pressures, from 250 to $495 \mathrm{GPa}$ [15-20], but the findings of these works are not yet unambiguously accepted by the entire scientific community.

To better understand the formation of superconductivity in hydrides at high transition temperatures, theory can provide valuable insight. It is widely accepted that the conventional electron-phonon mechanism is at play, being enhanced by the small ionic mass of hydrogen, the large electron-ion Coulomb interaction, and relatively weak electron-electron interaction. Although the appearance of superconductivity has not yet been reported, first-principles crystal structure investigations have determined that atomic hydrogen will adopt the $I 4_{1} /$ amd structure for a large pressure interval of 500-1000 GPa [21-23]. Advanced quantum Monte Carlo calculations estimated the transition pressure of $374 \mathrm{GPa}$ for the transition from the molecular phase to the atomic $I 4_{1} /$ amd phase [24]. Superconductivity in the latter phase has been investigated using first-principles electronic structure calculations of the electron and phonon bands and their coupling, using the semiempirical McMillan and Allen-Dynes equation $[22,25,26]$ or by solving the isotropic Eliashberg equations $[27,28]$. The obtained transition temperatures $T_{c}$ are around room temperature for a Coulomb pseudopotential value $\mu^{\star}=$ 0.10 . While anisotropic calculations of the superconducting state have been carried out for the molecular phase of hydrogen [29-31], such a study is yet elusive for atomic hydrogen in the $I 4_{1} /$ amd structure.

In this work we present a phonon-mode resolved analysis of metallic hydrogen in the superconducting state at pressures 
of 400 and $600 \mathrm{GPa}$, where the $I 4_{1} /$ amd phase is prevalent. Our calculations are carried out with the Uppsala Superconductivity (UPPSC) code [32-38]. Specifically, we solve here the anisotropic Migdal-Eliashberg equations using first-principles electron energies, phonon frequencies, and electron-phonon couplings as input. The total electron-phonon coupling constant $\lambda \simeq 2.32$ at $400 \mathrm{GPa}$ contains dominant contributions from the $B_{1 g}$ phonon mode, while the $A_{2 u}$ mode has the smallest impact. The remaining $E_{u}$ and $E_{g}$ modes both contribute with comparable and substantial magnitude to $\lambda$. We find $T_{c}$ approximately as room temperature for a reasonable range of Coulomb pseudopotential values $\mu^{\star}$, which is consistent with previous investigations $[25,27,28]$. Selectively investigating each of the phonon modes reveals that the $E_{u}$ mode contributes most to the $T_{c}$, despite having a subdominant role concerning the electron-phonon coupling strength. We provide a further proof of this observation by increasing the pressure to $600 \mathrm{GPa}$, where the critical temperature slightly increases, despite a reduction in electron-phonon coupling strength $\lambda \simeq 2.09$. In accordance with the just described picture, our mode-resolved Eliashberg calculations reveal that this stems from an enhanced contribution from the $E_{u}$ mode.

\section{METHODOLOGY}

\section{A. First-principles calculations}

We perform first-principles calculations within the density functional theory (DFT) framework using the QUANTUM ESPRESSO package [39]. We adopt the $I 4_{1} /$ amd crystal structure of atomic hydrogen that was predicted to be the stable structure over a large pressure range of 400-1000 GPa [22]. The exchange-correlation energy functional is treated within the generalized gradient corrected scheme of Perdew-BurkeErnzerhof [40]. The interactions between valence electrons and core are treated within the projector-augmented-wave (PAW) approach and the plane-wave basis set is constructed using an energy cutoff of $80 \mathrm{Ry}$. The Brillouin zone (BZ) integrations are carried out using a uniform dense $24 \times 24 \times 24$ Monkhorst-Pack k-point grid. The phonon dispersions and electron-phonon couplings are calculated on a dense $12 \times 12 \times 12$ q-point grid using density functional perturbation theory. All free parameters of the crystal lattice are optimized at 400 and $600 \mathrm{GPa}$. Since anharmonicity shows a minor effect on the critical temperature [28], these effects are not considered in the present calculations.

\section{B. Eliashberg theory calculations}

From $a b$ initio calculations we obtain branch $v$ and wave vector $\mathbf{q}$ dependent phonon frequencies $\omega_{\mathbf{q}, v}$, as well as electron-phonon coupling constants $\lambda_{\mathbf{q}, v}$ and quasiparticle lifetimes $\gamma_{\mathbf{q}, v}$. By defining bosonic Matsubara frequencies $q_{l}=2 \pi T l, l \in \mathbb{Z}$, at temperature $T$ we obtain the dynamic electron-phonon couplings via

$$
\lambda_{\mathbf{q}, l}=\sum_{\nu} \lambda_{\mathbf{q}, v} \frac{\omega_{\mathbf{q}, v}^{2}}{\omega_{\mathbf{q}, v}^{2}+q_{l}^{2}} .
$$

In the above we use the notation $g\left(\mathbf{q}, i q_{l}\right)=g_{\mathbf{q}, l}$ for any function $g$ for the sake of brevity. The couplings calculated from Eq. (1) serve as input for the self-consistent anisotropic Eliashberg equations

$$
\begin{aligned}
Z_{\mathbf{k}, m}=1+ & \frac{\pi T}{\omega_{m}} \sum_{\mathbf{k}^{\prime}, m^{\prime}} \frac{\delta\left(\xi_{\mathbf{k}^{\prime}}\right)}{N_{0}} \lambda_{\mathbf{k}-\mathbf{k}^{\prime}, m-m^{\prime}} \frac{\omega_{m^{\prime}}}{\sqrt{\omega_{m^{\prime}}^{2}+\Delta_{\mathbf{k}^{\prime}, m^{\prime}}^{2}}}, \\
\Delta_{\mathbf{k}, m}= & \frac{\pi T}{Z_{\mathbf{k}, m}} \sum_{\mathbf{k}^{\prime}, m^{\prime}} \frac{\delta\left(\xi_{\mathbf{k}^{\prime}}\right)}{N_{0}}\left[\lambda_{\mathbf{k}-\mathbf{k}^{\prime}, m-m^{\prime}}-\mu^{\star}\left(\omega_{c}\right)\right] \\
& \times \frac{\Delta_{\mathbf{k}^{\prime}, m^{\prime}}}{\sqrt{\omega_{m^{\prime}}^{2}+\Delta_{\mathbf{k}^{\prime}, m^{\prime}}^{2}}},
\end{aligned}
$$

describing the electron mass renormalization $Z_{\mathbf{k}, m}$ and superconducting gap function $\Delta_{\mathbf{k}, m}$ [33]. Again we write $f\left(\mathbf{k}, i \omega_{m}\right)=f_{\mathbf{k}, m}$, now with fermion Matsubara frequencies $\omega_{m}=\pi T(2 m+1), m \in \mathbb{Z}$. We use $\mu^{\star}$ as Anderson-Morel Coulomb pseudopotential, which enters Eq. (3) with a Matsubara frequency cutoff $\omega_{c}$. The critical temperature $T_{c}$ is defined as the smallest $T$ at which the self-consistent solution to Eqs. (2) and (3) yields a vanishing superconducting gap.

The electron density of states $N_{0}$ at the Fermi level is calculated via the adaptive smearing method, namely,

$$
N_{0}=\sum_{\mathbf{k}, n} \frac{1}{\sqrt{2 \pi}} \frac{1}{W_{\mathbf{k}, n}} \exp \left(-\frac{\xi_{\mathbf{k}, n}^{2}}{2 W_{\mathbf{k}, n}^{2}}\right),
$$

where the broadening tensor is defined as

$$
W_{\mathbf{k}, n}=a \Delta k\left|\frac{\partial \xi_{\mathbf{k}, n}}{\partial \mathbf{k}}\right|,
$$

in combination with the Methfessel-Paxton scheme [41]. In Eq. (5), $\Delta k$ is the momentum resolution and $a$ can be chosen $O(1)$ [42]. Furthermore, $\xi_{\mathbf{k}, n}$ is the electron dispersion as computed from DFT, with $\mathbf{k}$ a BZ momentum and $n$ a band index. We consider here only electronic states at the Fermi level, hence our calculations are carried out for the two partially occupied energy bands (shown further below).

We obtain a more simplified estimate of $T_{c}$ by employing the semiempirical McMillan equation [43], including a modification due to Allen and Dynes [44],

$$
T_{c}=\frac{\omega_{\log }}{1.2} \exp \left(\frac{-1.04(1+\lambda)}{\lambda\left(1-0.62 \mu^{\star}\right)-\mu^{\star}}\right) .
$$

Here $\lambda$ is the total electron-phonon coupling constant,

$$
\begin{aligned}
\lambda & =\sum_{\mathbf{q}, v} \lambda_{\mathbf{q}, v} \\
& =2 \int_{0}^{\infty} \frac{\alpha^{2} F(\omega)}{\omega} d \omega,
\end{aligned}
$$

and $\alpha^{2} F(\omega)$ is the real-frequency $\omega$ dependent Eliashberg function, given as

$$
\alpha^{2} F(\omega)=\frac{1}{2 \pi N_{0}} \sum_{\mathbf{q}, v} \delta\left(\omega-\omega_{\mathbf{q}, v}\right) \frac{\gamma_{\mathbf{q}, v}}{\omega_{\mathbf{q}, v}} .
$$

The characteristic phonon energy scale $\omega_{\log }$ is defined as

$$
\omega_{\log }=\exp \left[\frac{2}{\lambda} \int_{0}^{\infty} \frac{d \omega}{\omega} \alpha^{2} F(\omega) \log (\omega)\right] .
$$

As stated in the Introduction, atomic hydrogen metal gives rise to four different irreducible representations of the phonon 
modes. In the following Sec. III we present numerical results that partially correspond to only subsets of all these representations. This means that for an irreducible representation $\rho \in\left\{B_{1 g}, E_{g}, A_{2 u}, E_{u}\right\}$, any sum over branch index $v$ is to be understood as $\sum_{v \in \rho}$ [compare Eqs. (1), (7), and (9)]. The Eliashberg or McMillan equations are then solved for the resulting "partial" inputs.

\section{RESULTS}

We begin by calculating the electronic properties of metallic hydrogen in the optimized $I 4_{1} /$ amd structure. The results of our electronic structure calculations at a pressure of $400 \mathrm{GPa}$ are presented in Fig. 1. Figure 1(a) shows the computed electronic band structure plotted along high-symmetry directions in the BZ. The electronic states are highly dispersive forming very wide bands which reflects their nearly free electron nature. Two bands cross the Fermi level and are responsible for metallicity. One band corresponds to the bonding $s$ orbital and the other one to the antibonding $s$ orbital. The antibonding state is mostly unoccupied and crosses along the $\Gamma-Z$ direction. Our calculated electron band structure agrees well with previously reported results $[23,28,45]$.

The electronic density of states (DOS), shown in Fig. 1(b) with the orange color, is also consistent with the free electron behavior, being nearly parabolic below to Fermi level. Note that the DOS value at the Fermi level is higher than that of metallic molecular hydrogen in the $\mathrm{Cmca}-8$ phase [21], shown in green. Figure 1(c) shows the calculated three-dimensional Fermi surface of atomic hydrogen metal at $400 \mathrm{GPa}$, which consists of two sheets corresponding to the bonding and antibonding states. The bonding state leads to ribbonlike hole Fermi-surface sheets and the antibonding state leads to a concave lens shaped electron Fermi sheet at $Z$ (the Fermi surfaces were rendered using FERMISURFER software [46]). The sheets in Fig. 1(c) are colored according to the values of the Fermi velocities; the high Fermi velocities correspond to the free electron nature. The covalent character of the $\mathrm{H}-\mathrm{H}$ bonds is investigated by calculating the crystal orbital Hamiltonian population (COHP) functions [47-51] which counts the population of wave functions on two atomic orbitals of a pair of atoms [shown in Fig. 1(d)]. In a given energy window, negative values of COHP describe bonding interactions, whereas positive values of COHP describe antibonding interactions. This analysis shows that the overlap of nearest hydrogen states below the Fermi level are bonding states. The H-H bond in molecular phase (gray shaded area) has stronger covalent character than that of atomic phase. The integrated COHP values (computed with the code of Ref. [50]) are 1.30 and $3.27 \mathrm{eV} / \mathrm{H}-\mathrm{H}$ for atomic and molecular phases, respectively.

The computed phonon dispersions of metallic hydrogen at $400 \mathrm{GPa}$ (not shown) are very similar to the previously reported phonon dispersions [28].

After this we turn our attention to the superconducting properties of metallic atomic hydrogen. In Fig. 2(a) we show our convergence study of the global electron-phonon coupling strength $\lambda$, as obtained from Eq. (7), as a function of smearing $\sigma$, which is used by QUANTUM ESPRESSO to compute the electron-phonon coupling coefficients $\lambda_{\mathbf{q}, v}$. The results show
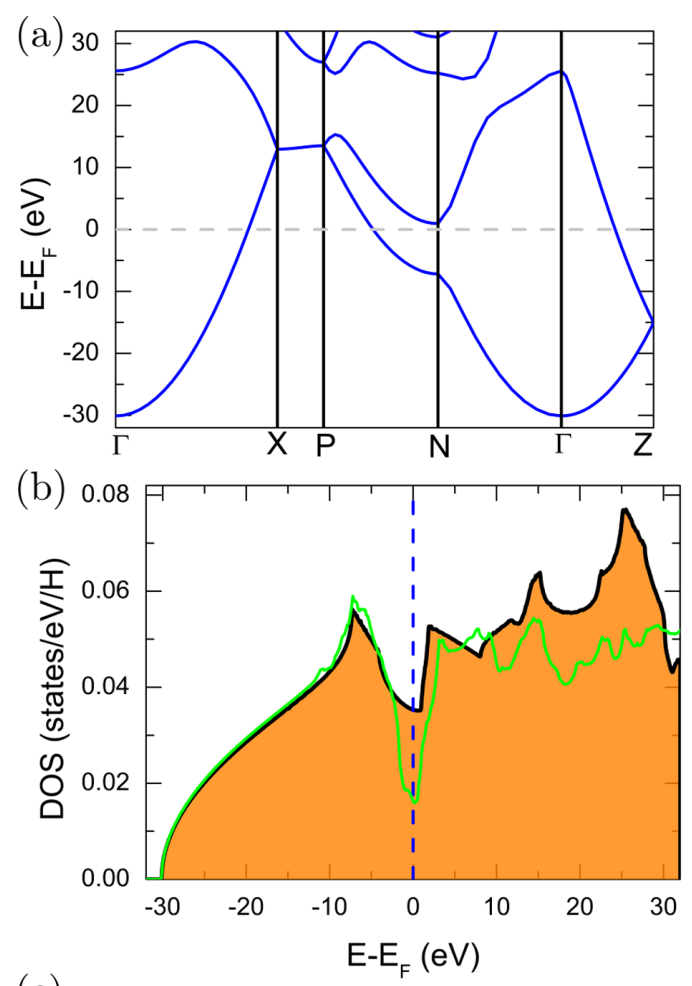

(c)
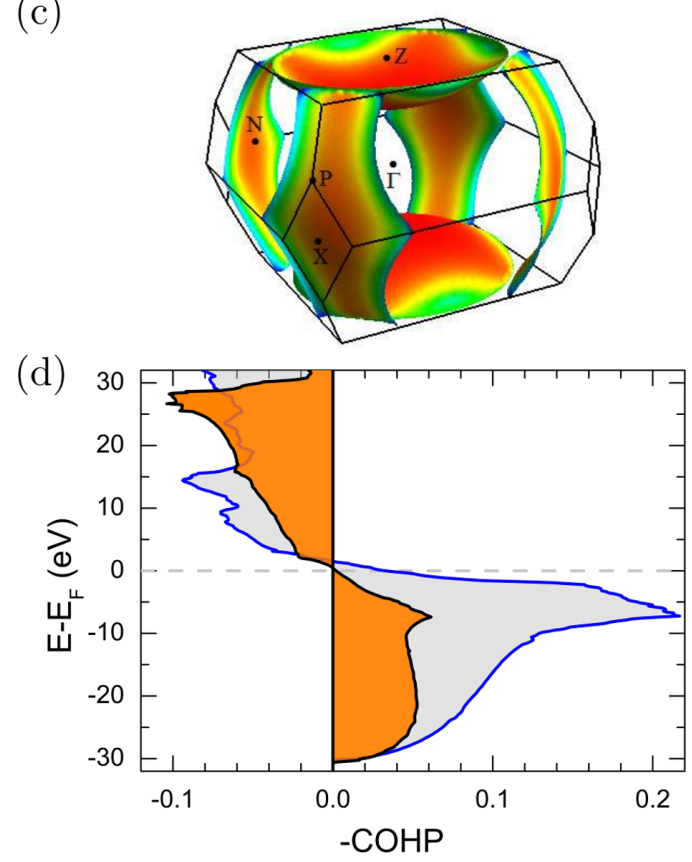

FIG. 1. Ab initio calculated electronic properties of metallic hydrogen at $400 \mathrm{GPa}$. (a) Electronic band structure along the high symmetry directions of the BZ. (b) Electronic density of states (DOS) function, shown in orange color. The light-green curve shows the DOS results of the Cmca-8 phase of molecular hydrogen [21]. (c) Computed Fermi-surface sheets, colored according to the Fermi velocity values, starting with blue (lowest velocities)-light-green (medium range velocities)-red colors (highest velocities). (d) The COHP functions. The orange shaded area shows the result for atomic hydrogen and the blue curve (gray shaded) shows the result for the Cmca-8 phase of molecular hydrogen. Negative COHP values represent bonding interactions and positive $\mathrm{COHP}$ values represent antibonding interactions. 
(a)

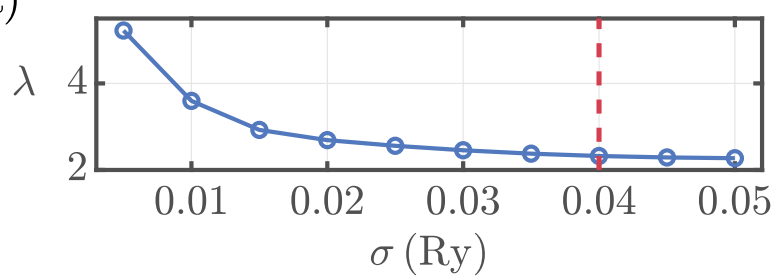

(b)

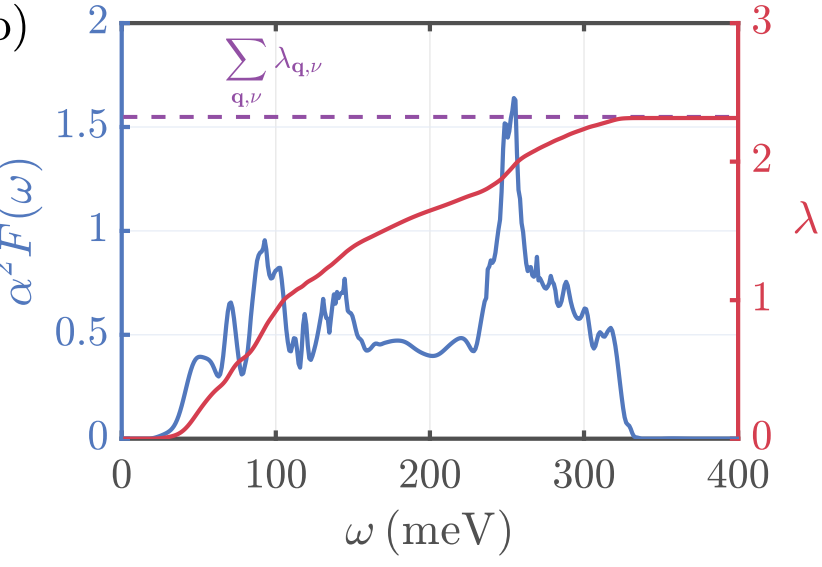

FIG. 2. (a) Global coupling constant as a function of broadening. (b) Frequency dependent Eliashberg function for a broadening value of $0.04 \mathrm{Ry}$ (blue curve). The red curve shows the cumulative coupling strength calculated from Eq. (8). The purple dashed line represents $\lambda$ as obtained from Eq. (7).

good convergence for $\sigma \gtrsim 0.04 \mathrm{Ry}$, and therefore this value of $\sigma$ will be used from here on. The converged value of $\lambda$ is 2.32 , which is compatible with the $\lambda=2.08$ computed for molecular hydrogen at $450 \mathrm{GPa}$ [29]. The coupling coefficient is also consistent with values for $\mathrm{H}_{3} \mathrm{~S}$ at $200 \mathrm{GPa}$ (2.19) [52], $\mathrm{LaH}_{10}$ at $250 \mathrm{GPa}$ (2.29) [4], and $\mathrm{YH}_{10}$ at $400 \mathrm{GPa}$ (2.41) [53]. Additionally, we have carefully checked that our results are properly converged in the electron and phonon momentum grids.

Now we turn to the analysis of how individual phonon modes contribute to the electron-phonon couplings. For this we calculate the Eliashberg function $\alpha^{2} F(\omega)$, shown in Fig. 2(b) in blue. The most prominent contributions appear at $\omega \sim 100 \mathrm{meV}$ and $\omega \sim 250 \mathrm{meV}$. This is further emphasized by the red curve representing the cumulative electron-phonon coupling as calculated from Eq. (8). The aforementioned frequencies lead to the steepest increase in $\lambda$ with $\omega$. As crosscheck, we calculated the total electron-phonon coupling using Eq. (7), shown in dashed purple. Both calculations yield identical, i.e., converged values of $\lambda$.

Next we solve the Eliashberg equations as a function of $T$ and pseudopotential $\mu^{\star}$, using the first-principles input computed for $\sigma=0.04 \mathrm{Ry}$. We show the result for the maximum superconducting gap $\Delta=\max _{\mathbf{k}} \Delta_{\mathbf{k}, m=0}$ in Fig. 3(a). In solid red we indicate the onset of superconductivity, hence the critical temperatures. Above we mention another recipe of calculating $T_{c}$ by means of the modified McMillan equation; the outcome is plotted as a dashed black line, using $\omega_{\mathrm{log}} \simeq 124 \mathrm{meV}$. We make the $\mu^{\star}$ dependence of $T_{c}$ explicit in Fig. 3(b), where we show the $T_{c}$ corresponding to room temperature in solid blue. As apparent, when using the modified
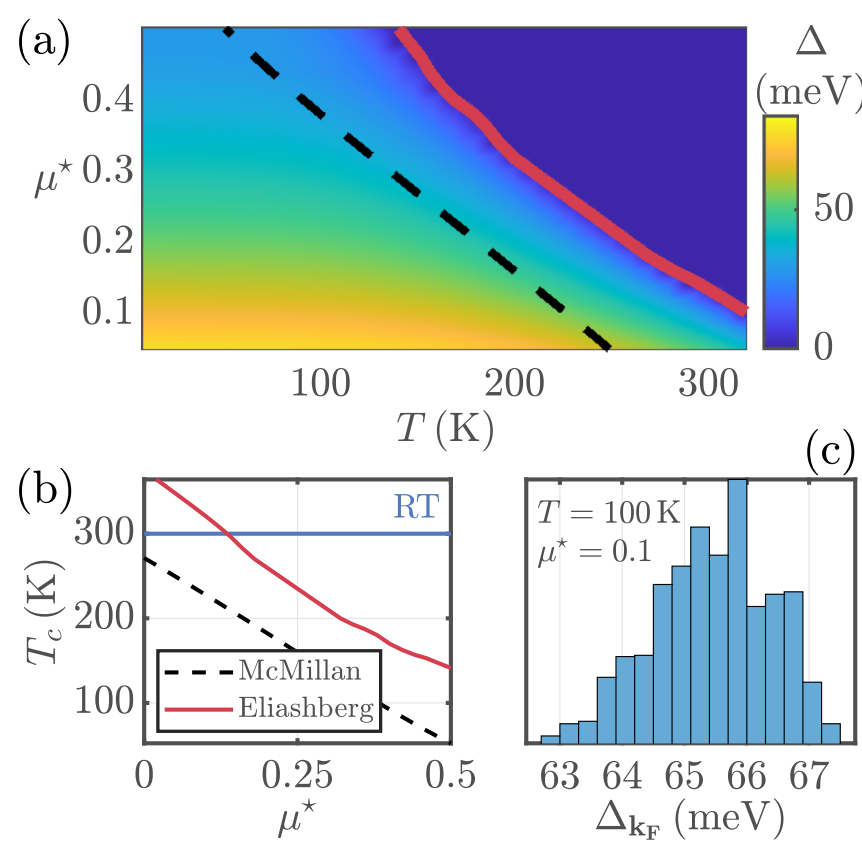

FIG. 3. (a) Self-consistently computed maximum superconducting gap for atomic hydrogen at $400 \mathrm{GPa}$ as a function of $T$ and screened Coulomb potential $\mu^{\star}$. The critical temperatures according to Eliashberg theory and the modified McMillan equation are drawn in solid red and dashed black lines, respectively. (b) Calculated critical temperature $T_{c}$ plotted against $\mu^{\star}$. (c) Computed momentum distribution of the gap $\Delta_{\mathbf{k}_{\mathbf{F}}}\left(=\Delta_{\mathbf{k}=\mathbf{k}_{\mathbf{F}}, m=0}\right)$ at $100 \mathrm{~K}$.

McMillan equation we underestimate the critical temperature, in comparison to the solution of the more accurate Eliashberg equations, for all values of $\mu^{\star}$. The dashed black line stays below room temperature even in the complete absence of pair-breaking Coulomb repulsion. The red solid line, on the other hand, predicts room-temperature superconductivity for values of $\mu^{\star}$ up to $\sim 0.14$. The underestimation of $T_{c}$ by the modified McMillan equation stems mainly from the strong electron-phonon coupling in atomic hydrogen. As has been reported in Ref. [44], estimating the critical temperature via the modified McMillan equation (6) becomes inaccurate as $\lambda$ exceeds 2, because the semiempirical equation (6) has been constructed for weak-coupling superconductors. The deviations in $T_{c}$ values computed with the McMillan equation can thus be significant, nearly $100 \mathrm{~K}$ [see Fig. 3(b) and Table I].

Here we model the superconducting state in metallic hydrogen via anisotropic Eliashberg theory, hence we have access to the momentum dependence of the superconducting gap. Analyzing our self-consistent solutions across the whole

TABLE I. Calculated transition temperatures for atomic hydrogen, using the modified McMillan equation, and isotropic and anisotropic Eliashberg theory.

\begin{tabular}{lcc}
\hline \hline & $T_{c}\left(\mu^{\star}=0.1\right)$ & $T_{c}\left(\mu^{\star}=0.2\right)$ \\
\hline Modified McMillan & $228 \mathrm{~K}$ & $183 \mathrm{~K}$ \\
Isotropic Eliashberg & $306 \mathrm{~K}$ & $250 \mathrm{~K}$ \\
Anisotropic Eliashberg & $321 \mathrm{~K}$ & $260 \mathrm{~K}$ \\
\hline \hline
\end{tabular}




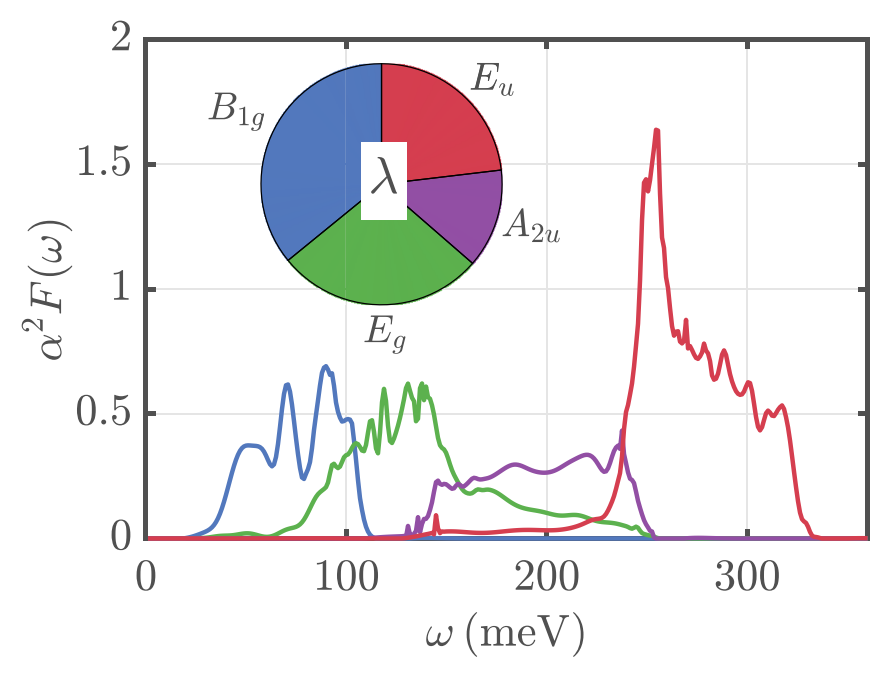

FIG. 4. Frequency dependent Eliashberg function of atomic hydrogen at $400 \mathrm{GPa}$ produced by the four different irreducible representations. The inset shows the contributions of $B_{1 g}, E_{g}, A_{2 u}$, and $E_{u}$ to the global electron-phonon coupling constant. The same colors are used for the main graph and the inset.

parameter ranges of $\mu^{\star}$ and $T$ [compare Fig. 3(a)] reveals that $\Delta_{\mathbf{k}_{\mathbf{F}}}\left(=\Delta_{\mathbf{k}=\mathbf{k}_{\mathrm{F}}, m=0}\right)$ is rather isotropic. As an explicit example, we show the momentum distribution of $\Delta_{\mathbf{k}_{\mathrm{F}}}$ in Fig. 3(c) for $T=100 \mathrm{~K}$ and $\mu^{\star}=0.1$. We find that the superconducting gap has a maximal variation of $\sim 5 \mathrm{meV}$ on the Fermi surface, which corresponds to an anisotropy of only around 7\% of the mean gap value. As a direct comparison we also computed $T_{c}$ for $\mu^{\star}=0.1$ and $\mu^{\star}=0.2$ with isotropic Eliashberg theory, the results of which are listed in Table I. As mentioned before, the strong electron-phonon coupling leads to a drastic underestimate of $T_{c}$ by the modified McMillan equation. Moreover, including the full $\mathrm{BZ}$ anisotropy leads to an additional increase in transition temperatures.

We now turn to the question about the most significant phonon branches. The irreducible representations in this system are $B_{1 g}$ (one mode), $E_{g}$ (two modes), $A_{2 u}$ (one mode), and $E_{u}$ (two modes). We split $\lambda_{\mathbf{q}, v}, \gamma_{\mathbf{q}, v}$, and $\omega_{\mathbf{q}, v}$ according to these subsets, and repeat the calculation of $\lambda$ and $\alpha^{2} F(\omega)$, respectively, via Eqs. (7) and (9). The relative contribution to the electron-phonon coupling due to the different phonon modes is shown as the inset in Fig. 4. In the main graph, we plot the partial Eliashberg functions arising from each irreducible representation. Concerning $\alpha^{2} F(\omega)$, we clearly see that each subset of phonon modes contributes mainly in a respective characteristic frequency range. As for the magnitude of $\lambda$, the largest (smallest) contributions are due to $B_{1 g}\left(A_{2 u}\right)$, while $E_{u}$ and $E_{g}$ are on a comparable intermediate level.

We want to examine how the different phonon modes affect the superconducting transition temperature. The $\mu^{\star}$ dependent result for $T_{c}$ as obtained from the full calculation is shown in Fig. 5(a) as a thick, light-red curve. The calculations are now repeated by selectively leaving out one particular subset of phonon modes, each corresponding to one of the irreducible representations. For example, the blue line in Fig. 5(a) is found by taking into account only the $E_{g}, A_{2 u}$, and $E_{u}$ irreducible representations, i.e., neglecting any influence due to
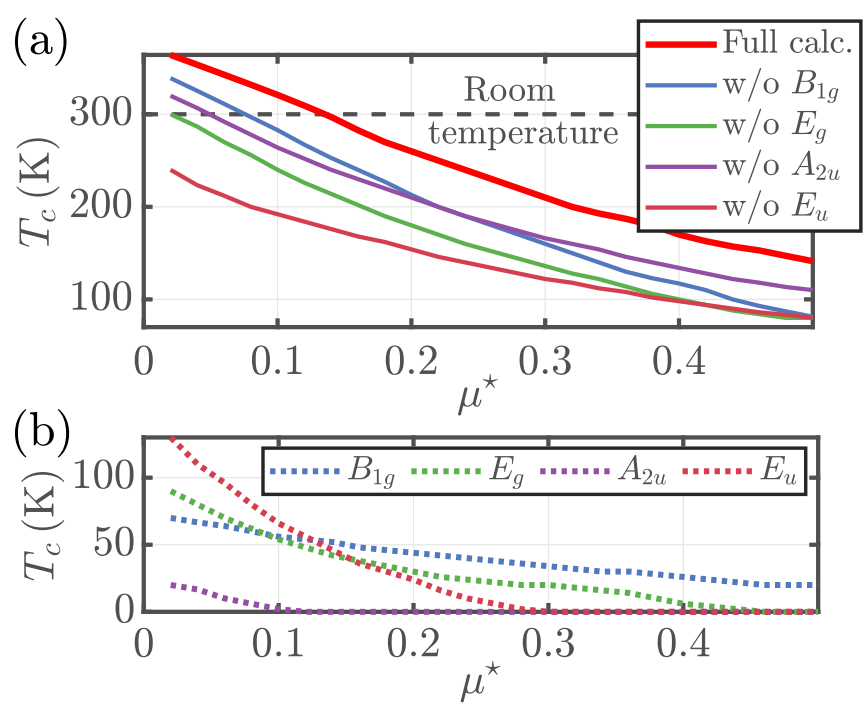

FIG. 5. Calculated transition temperatures as a function of the Coulomb pseudopotential. (a) The thick red line represents our result for including all modes in the system. The remaining four solid lines are found by neglecting one subset of phonon modes at a time (see legend). (b) Results computed for each individual irreducible representation.

$B_{1 g}$. From this we observe that the smallest decrease in $T_{c}$ is found when leaving out either the $B_{1 g}$ or the $A_{2 u}$ modes, hence their significance for superconductivity is comparatively minor. The largest loss in $T_{c}$ is found when excluding the $E_{u}$ modes (see the dark-red curve). Hence we conclude that phonon modes belonging to the $E_{u}$ representation are most important for the high-temperature superconducting state.

To investigate the branch dependence in more detail, we also performed calculations for each individual representation, the results of which are shown in Fig. 5(b) as dotted curves. The colors are chosen correspondingly to panel (a). In accordance to the discussion above, the largest critical temperatures are found for $E_{u}$ modes for $\mu^{\star} \lesssim 0.1$, with a maximum value of $T_{c} \sim 140 \mathrm{~K}$. For this particular representation the decrease in $T_{c}$ as a function of $\mu^{\star}$ is fastest, which we find to be due to the very isotropic nature of the corresponding partial coupling. On the other extreme, the decay in critical temperature when considering only the $B_{1 g}$ mode is relatively slow, which stems conversely from its rather anisotropic contribution to the electron-phonon coupling. Due to the highly nonlinear nature of Eliashberg theory, the total $T_{c}$ in atomic hydrogen is not simply the sum of critical temperatures as obtained by considering each irreducible representation in an isolated way. This can be easily seen by comparing the full calculation in Fig. 5(a) for large values of $\mu^{\star}$ with the corresponding curves in Fig. 5(b).

We performed similar calculations for atomic hydrogen at $600 \mathrm{GPa}$. As shown in Fig. 6(a), we find a slight decrease in the value of electron-phonon coupling, $\lambda=2.09$, but overall, as can be seen in Fig. 6(b), $T_{c}$ increases slightly. Similarly, a higher critical temperature than for $400 \mathrm{GPa}$ is found when considering the modified McMillan equation, where the characteristic phonon energy scale for $600 \mathrm{GPa}$ is $\omega_{\log } \simeq 141 \mathrm{meV}$. Although this behavior may seem 


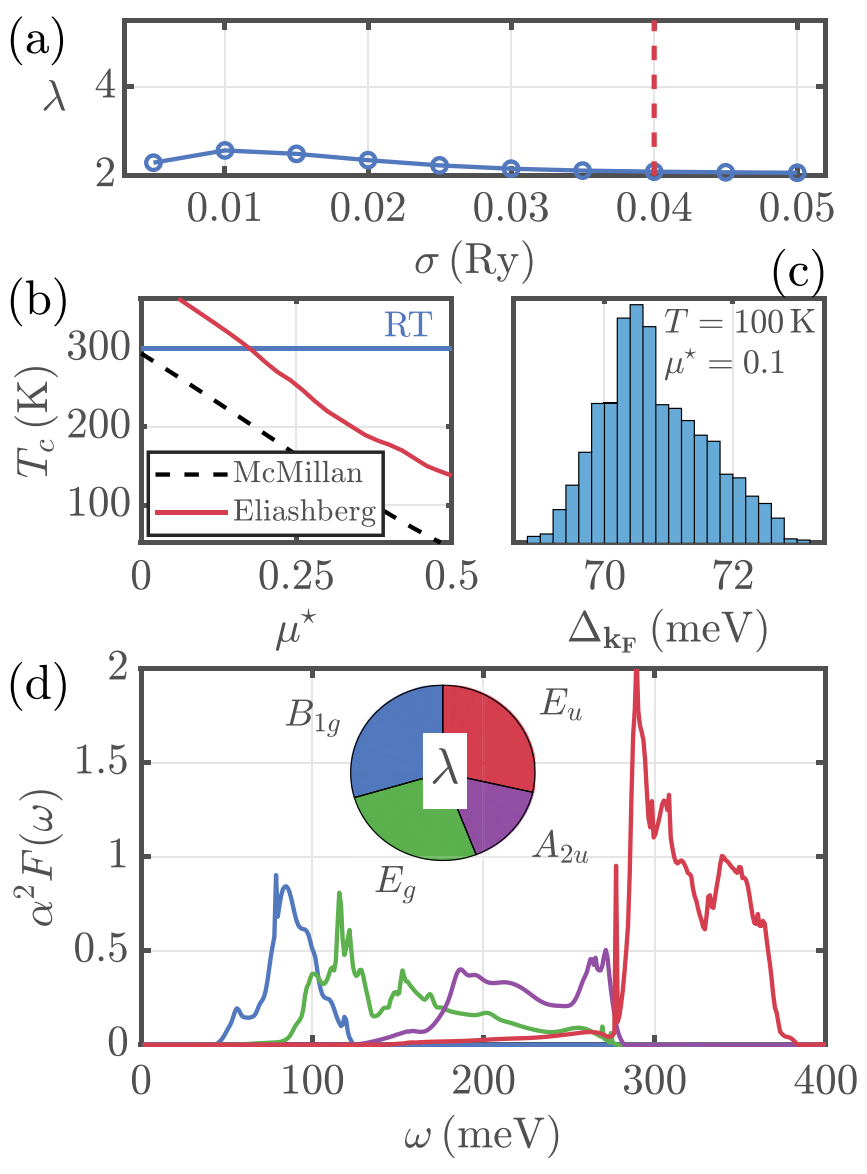

FIG. 6. (a) Computed global coupling constant $\lambda$ as a function of broadening for atomic hydrogen at a pressure of $600 \mathrm{GPa}$. (b) The critical temperature versus Coulomb pseudopotential $\mu^{\star}$ computed with Eliashberg theory and with the modified McMillan equation. (c) Momentum distribution of the superconducting gap on the Fermi surface. (d) As Fig. 4, but for atomic hydrogen at a pressure of $600 \mathrm{GPa}$.

counterintuitive, it can be explained by the way different phonon modes contribute to superconductivity. Despite the small decrease in the total electron-phonon coupling, we now find an increased coupling to $E_{u}$ symmetry modes, as illustrated in the inset of Fig. 6(d), which leads to an increase in $\omega_{\log }$ and therefore to the increase in transition temperature. Hence, this underlines the dominant contribution stemming from the $E_{u}$ phonon modes. Similarly as before, we show the computed momentum distribution of $\Delta_{\mathbf{k}_{\mathbf{F}}}$ for $T=100 \mathrm{~K}$ and $\mu^{\star}=0.1$ in Fig. 6(c). As can be recognized, with a range of approximately $4 \mathrm{meV}$, the superconducting gap becomes even more isotropic at increasing pressures.

\section{CONCLUSIONS}

In summary, we have reported a detailed analysis of the superconducting properties of metallic atomic hydrogen under high pressure conditions. To this end, we solved the anisotropic Migdal-Eliashberg equations, in combination with first-principles input results for the electron energies, phonons, and electron-phonon couplings. Our calculations show that, although the $\mathrm{H}-\mathrm{H}$ covalent bond has weakened in atomic hydrogen metal as compared to the molecular hydrogen phase, it still has a substantial amount of covalent character. Further, we find that metallic atomic hydrogen exhibits above room-temperature superconductivity for reasonable values of the screened Coulomb pseudopotential $\mu^{\star}$. Analyzing which modes contribute most, we find that the high transition temperature is mainly due to the $E_{u}$ phonon modes. The critical transition temperature shows only a slight increase with pressure. Computing explicitly the momentum anisotropy of the superconducting gap, we obtain a relatively small anisotropy of about $7 \%$ at $100 \mathrm{~K}$ and $400 \mathrm{GPa}$.

\section{ACKNOWLEDGMENTS}

A.K.V. and P.M. acknowledge the support of ANUPAM supercomputing facility of BARC. F.S., A.A., and P.M.O. acknowledge support by the Swedish Research Council (VR), the Röntgen-Ångström Cluster, the Knut and Alice Wallenberg Foundation (Grant No. 2015.0060), and the Swedish National Infrastructure for Computing (SNIC).
[1] C. J. Pickard, I. Errea, and M. I. Eremets, Ann Rev. Condens. Matter Phys. 11, 57 (2020).

[2] J. A. Flores-Livas, L. Boeri, A. Sanna, G. Profeta, R. Arita, and M. Eremets, Phys. Rep. 856, 1 (2020).

[3] A. P. Drozdov, M. I. Eremets, I. A. Troyan, V. Ksenofontov, and S. I. Shylin, Nature (London) 525, 73 (2015).

[4] H. Liu, I. I. Naumov, R. Hoffmann, N. W. Ashcroft, and R. J. Hemley, Proc. Natl. Acad. Sci. USA 114, 6990 (2017).

[5] A. P. Drozdov, P. P. Kong, V. S. Minkov, S. P. Besedin, M. A. Kuzovnikov, S. Mozaffari, L. Balicas, F. Balakirev, D. Graf, V. B. Prakapenka, E. Greenberg, D. A. Knyazev, M. Tkacz, and M. I. Eremets, Nature (London) 569, 528 (2019).

[6] M. Somayazulu, M. Ahart, A. K. Mishra, Z. M. Geballe, M. Baldini, Y. Meng, V. V. Struzhkin, and R. J. Hemley, Phys. Rev. Lett. 122, 027001 (2019).
[7] I. A. Troyan, D. V. Semenok, A. G. Kvashnin, A. V. Sadakov, O. A. Sobolevskiy, V. M. Pudalov, A. G. Ivanova, V. B. Prakapenka, E. Greenberg, A. G. Gavriliuk, V. V. Struzhkin, A. Bergara, I. Errea, R. Bianco, M. Calandra, F. Mauri, L. Monacelli, R. Akashi, and A. R. Oganov, arXiv:1908.01534.

[8] P. P. Kong, V. S. Minkov, M. A. Kuzovnikov, S. P. Besedin, A. P. Drozdov, S. Mozaffari, L. Balicas, F. F. Balakirev, V. B. Prakapenka, E. Greenberg, D. A. Knyazev, and M. I. Eremets, arXiv:1909.10482.

[9] E. Snider, N. Dasenbrock-Gammon, R. McBride, M. Debessai, H. Vindana, K. Vencatasamy, K. V. Lawler, A. Salamat, and R. P. Dias, Nature (London) 586, 373 (2020).

[10] A. D. Grockowiak, M. Ahart, T. Helm, W. A. Coniglio, R. Kumar, M. Somayazulu, Y. Meng, M. Oliff, V. Williams, N. W. Ashcroft, R. J. Hemley, and S. W. Tozer, arXiv:2006.03004. 
[11] N. W. Ashcroft, Phys. Rev. Lett. 21, 1748 (1968).

[12] E. Wigner and H. B. Huntington, J. Chem. Phys. 3, 764 (1935).

[13] H.-K. Mao and R. J. Hemley, Rev. Mod. Phys. 66, 671 (1994).

[14] J. M. McMahon, M. A. Morales, C. Pierleoni, and D. M. Ceperley, Rev. Mod. Phys. 84, 1607 (2012).

[15] H. K. Mao and R. J. Hemley, Science 244, 1462 (1989).

[16] A. F. Goncharov, E. Gregoryanz, R. J. Hemley, and H.-K. Mao, Proc. Natl. Acad. Sci. USA 98, 14234 (2001).

[17] M. I. Eremets, I. A. Troyan, and A. P. Drozdov, arXiv: 1601.04479.

[18] R. P. Dias and I. F. Silvera, Science 355, 715 (2017).

[19] C. Ji, B. Li, W. Liu, J. S. Smith, A. Majumdar, W. Luo, R. Ahuja, J. Shu, J. Wang, S. Sinogeikin, Y. Meng, V. B. Prakapenka, E. Greenberg, R. Xu, X. Huang, W. Yang, G. Shen, W. L. Mao, and H.-K. Mao, Nature (London) 573, 558 (2019).

[20] P. Loubeyre, F. Occelli, and P. Dumas, Nature (London) 577, 631 (2020).

[21] C. J. Pickard and R. J. Needs, Nat. Phys. 3, 473 (2007).

[22] J. M. McMahon and D. M. Ceperley, Phys. Rev. Lett. 106, 165302 (2011).

[23] N. N. Degtyarenko and E. A. Mazur, JETP Lett. 104, 319 (2016).

[24] S. Azadi, B. Monserrat, W. M. C. Foulkes, and R. J. Needs, Phys. Rev. Lett. 112, 165501 (2014).

[25] J. M. McMahon and D. M. Ceperley, Phys. Rev. B 85, 219902(E) (2012).

[26] Y. Yan, J. Gong, and Y. Liu, Phys. Lett. A 375, 1264 (2011).

[27] A. P. Durajski, R. Szcześniak, and A. M. Duda, Solid State Commun. 195, 55 (2014).

[28] M. Borinaga, I. Errea, M. Calandra, F. Mauri, and A. Bergara, Phys. Rev. B 93, 174308 (2016).

[29] P. Cudazzo, G. Profeta, A. Sanna, A. Floris, A. Continenza, S. Massidda, and E. K. U. Gross, Phys. Rev. Lett. 100, 257001 (2008)

[30] P. Cudazzo, G. Profeta, A. Sanna, A. Floris, A. Continenza, S. Massidda, and E. K. U. Gross, Phys. Rev. B 81, 134505 (2010).

[31] P. Cudazzo, G. Profeta, A. Sanna, A. Floris, A. Continenza, S. Massidda, and E. K. U. Gross, Phys. Rev. B 81, 134506 (2010).

[32] The Uppsala Superconductivity (UPPSC) code provides a package to self-consistently solve the anisotropic, multiband, and full-bandwidth Eliashberg equations for frequency-even and odd superconductivity mediated by phonons, charge, or spin fluctuations on the basis of ab initio calculated input.

[33] A. Aperis, P. Maldonado, and P. M. Oppeneer, Phys. Rev. B 92, 054516 (2015).

[34] F. Schrodi, A. Aperis, and P. M. Oppeneer, Phys. Rev. B 98, 094509 (2018).

[35] F. Schrodi, A. Aperis, and P. M. Oppeneer, Phys. Rev. B 99 , 184508 (2019).

[36] F. Schrodi, P. M. Oppeneer, and A. Aperis, Phys. Rev. B 102, 024503 (2020).

[37] F. Schrodi, A. Aperis, and P. M. Oppeneer, Phys. Rev. B 102, 014502 (2020).

[38] F. Schrodi, A. Aperis, and P. M. Oppeneer, Phys. Rev. B 102, 180501(R) (2020).

[39] P. Giannozzi et al., J. Phys.: Condens. Matter 21, 395502 (2009).

[40] J. P. Perdew, K. Burke, and M. Ernzerhof, Phys. Rev. Lett. 77, 3865 (1996).

[41] M. Methfessel and A. T. Paxton, Phys. Rev. B 40, 3616 (1989).

[42] J. R. Yates, X. Wang, D. Vanderbilt, and I. Souza, Phys. Rev. B 75, 195121 (2007).

[43] W. L. McMillan, Phys. Rev. 167, 331 (1968).

[44] P. B. Allen and R. C. Dynes, Phys. Rev. B 12, 905 (1975).

[45] N. A. Kudryashov, A. A. Kutukov, and E. A. Mazur, JETP Lett. 105, 430 (2017).

[46] M. Kawamura, Comput. Phys. Commun. 239, 197 (2019).

[47] R. Dronskowski and P. E. Blöchl, J. Phys. Chem. 97, 8617 (1993).

[48] A. Grechnev, R. Ahuja, and O. Eriksson, J. Phys.: Condens. Matter 15, 7751 (2003).

[49] S. Steinberg and R. Dronskowski, Crystals 8, 225 (2018).

[50] F. Boucher, O. Jepsen, and O. K. Andersen, Stuttgart tightbinding LMTO program version 4.7, Max-Planck-Institut für Festkorperförschung, Stuttgart, Federal Republic of Germany.

[51] A. K. Verma and P. Modak, Phys. Chem. Chem. Phys. 20, 26344 (2018).

[52] D. Duan, Y. Liu, F. Tian, D. Li, X. Huang, Z. Zhao, H. Yu, B. Liu, W. Tian, and T. Cui, Sci. Rep. 4, 6968 (2014).

[53] F. Peng, Y. Sun, C. J. Pickard, R. J. Needs, Q. Wu, and Y. Ma, Phys. Rev. Lett. 119, 107001 (2017). 